\title{
Microscopic Understanding of the Carrier Transport Process in Ge Nanocrystals Films
}

\author{
Dan Shan $\mathbb{D}^{1,2}$ Hongyu Wang, ${ }^{2,3}$ Mingjun Tang, ${ }^{1}$ and Jun Xu $\mathbb{D}^{2}$ \\ ${ }^{1}$ College of Electric and Information Technology, Yangzhou Polytechnic Institute, Yangzhou 225127, China \\ ${ }^{2}$ National Laboratory of Solid State Microstructures and School of Electronic Science and Engineering and Collaborative Innovation \\ Center of Advanced Microstructures, Nanjing University, Nanjing 210093, China \\ ${ }^{3}$ Tongda College, Nanjing University of Posts and Telecommunications, Nanjing 210003, China
}

Correspondence should be addressed to Jun Xu; junxu@nju.edu.cn

Received 18 January 2018; Revised 16 March 2018; Accepted 26 March 2018; Published 30 May 2018

Academic Editor: P. Davide Cozzoli

Copyright (c) 2018 Dan Shan et al. This is an open access article distributed under the Creative Commons Attribution License, which permits unrestricted use, distribution, and reproduction in any medium, provided the original work is properly cited.

Hydrogenated amorphous germanium ( $a-\mathrm{Ge}: \mathrm{H})$ films were prepared by a plasma enhanced chemical vapor deposition (PECVD) technique. Ge nanocrystals (Ge NCs) films were obtained by thermal annealing of the as-deposited samples at various temperatures. P-type behavior in Ge NCs films without any external doping was attributed to the holes accumulation caused by acceptor-like surface states. It can be found that the dark conductivity and Hall mobility reached as high as $25.6 \mathrm{~S} / \mathrm{cm}$ and $182 \mathrm{~cm} / \mathrm{V} \cdot \mathrm{s}$ in the Ge NCs film annealed at $500^{\circ} \mathrm{C}$, which were increased by over four and three orders of magnitude higher than that of the as-deposited film $\left(1.3 \times 10^{-3} \mathrm{~S} / \mathrm{cm}\right.$ and $0.14 \mathrm{~cm}^{2} / \mathrm{V} \cdot \mathrm{s}$, resp.). Carrier transport mechanisms of Ge NCs films association with the microstructural characteristics were investigated. Three kinds of temperature-dependent conductivity behaviors, which exhibit the linear relationships of $\ln \sigma$ versus $T^{-1 / 4}, T^{-1 / 2}$, and $T^{-1}$, respectively, were observed in the temperature regions from $10 \mathrm{~K}$ to $500 \mathrm{~K}$, showing different microscopic mechanisms governing carrier transport in Ge NCs film.

\section{Introduction}

Silica-based semiconductor nanocrystals have attracted much interest in recent years due to their possible applications in many kinds of nanoelectronic and optoelectronic devices such as next generation of solar cells, nonvolatile memories, and single electron transistors [1-5]. Compared with $\mathrm{Si}$, Ge has larger electron and hole mobility, which can be used to fabricate the Ge-based thin film transistor (TFT) and nonvolatile memories with good device performance $[6,7]$. Furthermore, Ge has a narrower band-gap $(0.67 \mathrm{eV})$ and high phonon responsivity in the near-infrared region, so it is suited to many near-infrared applications $[8,9]$. In order to further improve the device performance, detailed knowledge of transport mechanisms across these nanocrystals becomes necessary and is considered indispensable.

Studies of Si NCs films have provided a certain insight into the electronic characteristics of such material. At the previous works, there were many transport mechanisms applied to describe in nanocrystals, such as thermal activated transport in the extended states $[10,11]$, tunneling through the grain boundary (GB) potential barriers [12, 13], Mott variable-range hoping mechanism [14], percolation-hopping transport mechanism $[15,16]$, space charge limited currents, and single electron effects $[17,18]$. It looked like that the transport process was strongly influenced by the film structures and compositions. The actual conduction mechanisms in such composites with different nanostructured semiconductors remain a dominantly open question.

In the present work, we have investigated the microstructures and carrier transport properties of Ge NCs films. It was found that Ge NCs were formed in films by annealing the amorphous samples and the average grain size was sequentially increased by further increasing the annealing temperature. Both of the dark conductivity and Hall mobility were significantly enhanced after annealing. A p-type behavior of Ge NCs films without intentional doping was observed, indicating inherent holes generation in the structure. Furthermore, temperature-dependent carrier transport processes in Ge NCs films were systematically studied in 
the temperature range of $10-500 \mathrm{~K}$. It was found that three different mechanisms dominated the carrier transport process, respectively, in different temperature regions. The possible microscopic mechanisms governing charge transport were briefly discussed.

\section{Experiment}

The hydrogenated amorphous germanium ( $a-\mathrm{Ge}: \mathrm{H})$ films were prepared by a plasma enhanced chemical vapor deposition (PECVD) system using a gas of pure germane $\left(\mathrm{GeH}_{4}\right)$ with a flow rate of 5 SCCM (SCCM denotes standard cubic centimeter per minute). During the growth process, the gaschamber pressure and substrate temperature were $10 \mathrm{mTorr}$ and $250^{\circ} \mathrm{C}$ using $30 \mathrm{~W}$ of radio-frequency power, respectively. The films thickness is about $150 \mathrm{~nm}$. After deposition, the films were subsequently annealed in a conventional furnace at temperatures of 400 and $500^{\circ} \mathrm{C}$ for $1 \mathrm{~h}$ in nitrogen ambient. Quartz plates and monocrystalline Si wafers were used as substrates for the various measurements.

The microstructures of the films before and after annealing were measured using Raman (model: HR800, Jobin Yvon Horiba Inc.). The high-resolution transmission electron microscopy (TEM) images were observed by a TECNAI G2F20 FEI high-resolution transmission electron microscopy. The optical band-gap was deduced by Tauc plots based on the optical absorption spectra measured by using a Shimadzu UV-3600 spectrophotometer. The dark conductivity and Hall mobility were obtained by temperaturedependent Hall measurements using van der Pauw (VDP) geometry with the LakeShore 8400 Hall effect measurement system. The temperature of Hall measurement ranges from $10 \mathrm{~K}$ to $500 \mathrm{~K}$. The samples were prepared with coplanar $\mathrm{Al}$ electrodes on the four corners of the film by vacuum thermal evaporation followed by a $400^{\circ} \mathrm{C}$ alloying treatment of 30 mins to achieve the ohmic contacts which is confirmed by the linear current-voltage relationship.

\section{Results and Discussion}

Raman spectra of Ge NCs films annealed at various temperatures are shown in Figure 1. As a reference, the Raman spectrum of film before annealing is also plotted. The signals at 273 and $300 \mathrm{~cm}^{-1}$ represent the transverse-optical (TO) vibration mode of amorphous $\mathrm{Ge}$ and the TO vibration mode of the crystalline $\mathrm{Ge}$, respectively. A sharp and strong Raman peak close to $300 \mathrm{~cm}^{-1}$ can be clearly identified for all the films after annealing, which indicates the crystallization of amorphous Ge films. The crystalline volume fraction $\left(X_{c}\right)$ is estimated according to the formula: $X_{c}=I_{c} /\left(I_{c}+I_{a}\right)$ [19], where $I_{c}$ and $I_{a}$ are the crystalline and amorphous part of the integrated Raman scattering intensity, respectively. It was found that $X_{c}$ was about $63 \%$ for the Ge NCs film annealed at $400^{\circ} \mathrm{C}$ and was gradually increased to nearly $80 \%$ after annealing at $500^{\circ} \mathrm{C}$. The size of Ge NCs $(R)$ annealed at various temperatures can be roughly estimated from the shift between $\omega_{1}$ and $\omega_{\text {LO-TO }}$ using the bending parameters of bulk optical phonon dispersion curve $\beta_{\mathrm{LO}-\mathrm{TO}}$, according to the

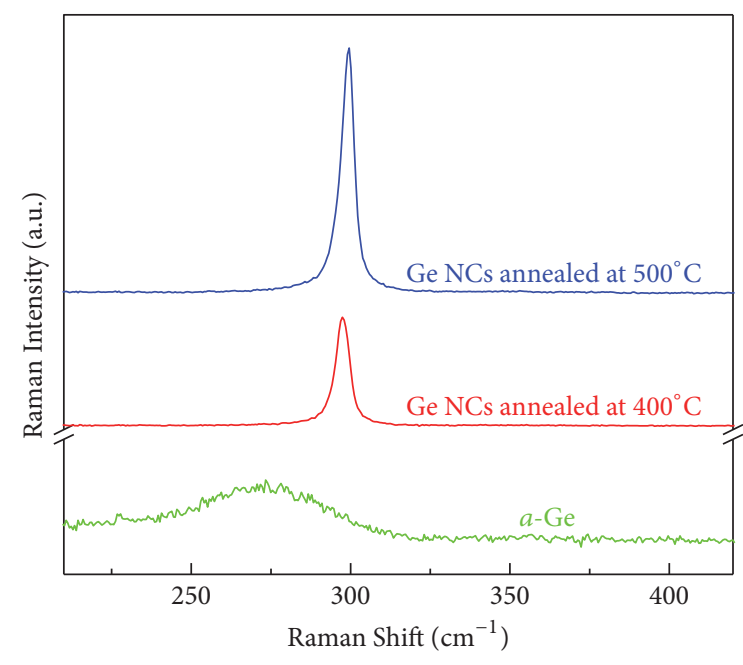

FIGURE 1: Raman spectra of the as-deposited sample and samples annealed at various temperature.

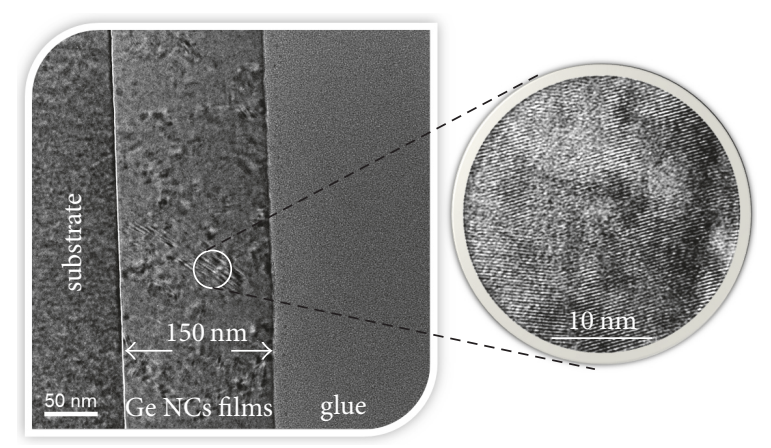

Figure 2: The cross-sectional TEM image of the Ge NCs film annealed at $500^{\circ} \mathrm{C}$; the inset is the high-resolution TEM image.

formula $\omega_{1}^{2}=\omega_{\mathrm{LO}-\mathrm{TO}}^{2}-\beta_{\mathrm{LO}-\mathrm{TO}}(\pi / R)^{2}$, where $\omega_{1}$ and $\omega_{\mathrm{LO}-\mathrm{TO}}$ are the Raman spectra of Ge NCs films and bulk Ge, respectively [20]. It was worth noting that the average sizes were about $7 \mathrm{~nm}$ and $17 \mathrm{~nm}$ for the Ge NCs films annealed at 400 and $500^{\circ} \mathrm{C}$, which indicates that the higher temperature annealing can increase the grain size of Ge NCs.

The microstructures of Ge NCs films were investigated using the cross-sectional TEM observations. Figure 2 shows the cross-sectional TEM images of the Ge NCs film annealed at $500^{\circ} \mathrm{C}$. The thickness of film is about $150 \mathrm{~nm}$, which is in good agreement with the predesigned value estimated by the deposition rate. The formation of Ge NCs with various orientations can be further identified in the high-resolution TEM image as given in the inset. A large number of Ge NCs with the grain sizes of more than $10 \mathrm{~nm}$ spread over the film, indicating a good crystallization of the Ge NCs film. It is also shown that some nanoparticles exhibit the elliptic shapes with the vertical sizes of $10 \mathrm{~nm}$ and the lateral sizes as large as $20 \mathrm{~nm}$.

The optical band-gap $E_{g}$, which is usually used to describe the light absorption in amorphous and nanocrystalline semiconductor films $[21,22]$, was deduced by using the Tauc 


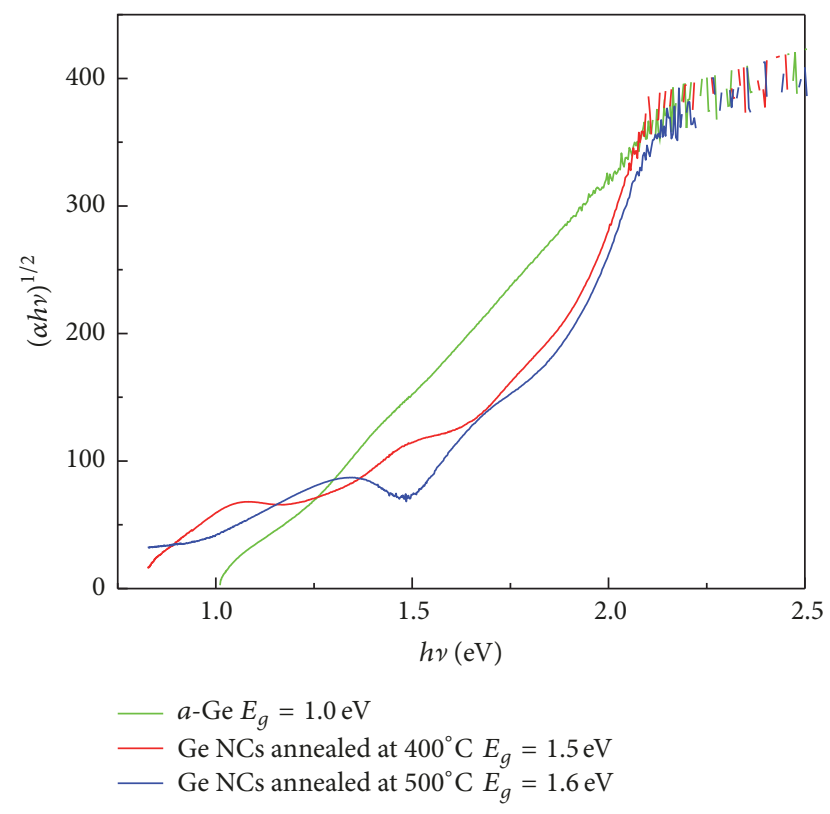

FIgURE 3: Tauc's plot of $(\alpha h \nu)^{1 / 2}$ versus photon energy $h v$ for asdeposited film and films annealed at 400 and $500^{\circ} \mathrm{C}$.

plot. Figure 3 shows Tauc's plot of $(\alpha h \nu)^{1 / 2}$ versus photon energy $h \nu$ for as-deposited film and films annealed at 400 and $500^{\circ} \mathrm{C}$. The optical band-gap is $1.0 \mathrm{eV}$ for the as-deposited film, which represents the band-gap of amorphous Ge film. After annealing, the value of $E_{g}$ was increased and reached 1.5 and $1.6 \mathrm{eV}$ for the films annealed at 400 and $500^{\circ} \mathrm{C}$. As we know, the structure of films is changed from amorphous phases to nanocrystalline phases during the thermal annealing. For the crystallized sample, the disordered grain boundary regions, which have a higher optical band-gap compared to the amorphous and nanocrystalline regions, are widely distributed in Ge NCs films, leading to a prevalent contribution to the overall optical gap [23]. Therefore, the increase of optical band-gap for the annealed films may be ascribed to the increase of nanocrystalline components in films. Another possibility is the incorporation of $\mathrm{N}$ or $\mathrm{O}$ into the annealed films during the annealing process, which may also result in the increase of optical band-gap.

The electrical characterizations of all the films were performed applying the temperature-dependent Hall measurements. Figure 4 shows the dark conductivities and Hall mobilities measured at room temperature for the asdeposited film and films after annealing. It was interesting to find that Hall mobilities were significantly increased from $0.14 \mathrm{~cm}^{2} / \mathrm{V}$.s for the as-deposited film to $182 \mathrm{~cm}^{2} / \mathrm{V}$.s for the Ge NCs film annealed at $500^{\circ} \mathrm{C}$, which are improved more than three orders of magnitude after annealing. Compared with any other work investigating the electronic properties of Ge NCs, our values of Hall mobility are more outstanding than those of previous reports $[24,25]$. It is well known that the enhanced Hall mobility after annealing is actually attributed to the formation of Ge NCs in the films [12]. Thus, the highlighted Hall mobilities further reveal a quality crystallization of Ge NCs films via thermal annealing in our

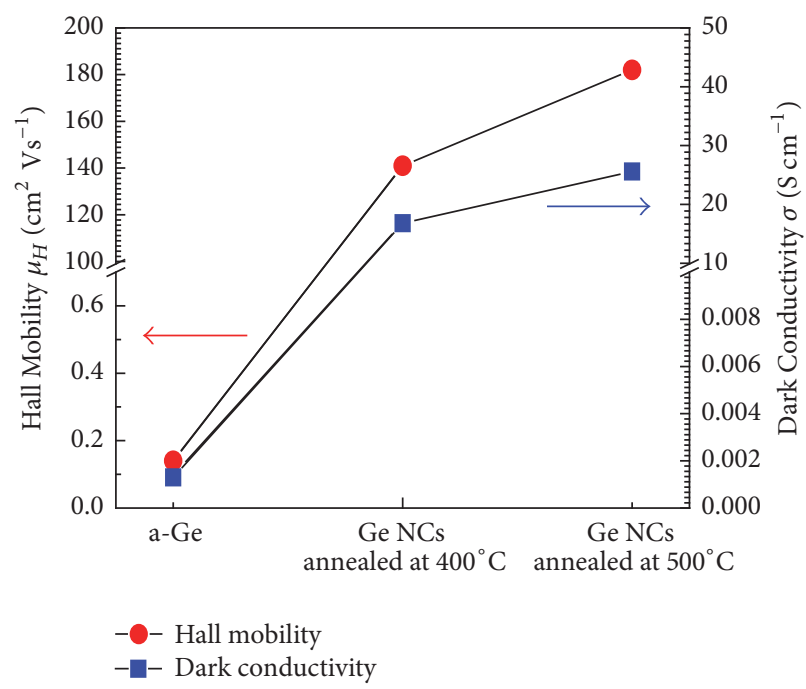

Figure 4: Room temperature dark conductivities and Hall mobilities for as-deposited film and films after annealing.

recent work. Meanwhile, the conductivity of as-deposited film was found to be $1.3 \times 10^{-3} \mathrm{~S} / \mathrm{cm}$. Thermal annealing further improved the films conductivities. For the Ge NCs film annealed at $500^{\circ} \mathrm{C}$, a value as high as $25.6 \mathrm{~S} / \mathrm{cm}$ was recorded, which is much higher than the previously reported data $[25,26]$. As we know, the conductivity is usually determined by the carrier concentration and Hall mobility. As a high Hall mobility discussed above, a p-type behavior with holes concentration of more than $10^{17} / \mathrm{cm}^{3}$ order was interestingly presented in the Ge NCs films without intentional doping. Zhang et al. investigated the electronic properties of Ge NCs embedded in $\mathrm{SiO}_{2}$ matrix and also found a p-type behavior in the films. They suggested that this p-type behavior in the undoped Ge NCs films was attributed to the deep-acceptorlike surface states [25], which was usually observed in Ge nanowires and $\mathrm{Ge} / \mathrm{Si}$ Core-Shell nanowires $[27,28]$. It is known that Ge NCs films usually have a charge neutrality level lying close to the valence band due to the asymmetrical distribution of surface state effective density [29]. Therefore, fixed negative charges are built up at the surfaces due to the occupation of dominated deep-acceptor-like surface states, which are usually related to the dangling bonds [30, 31]. As a consequence, the energy bands near the surface tend to bend up and attract extra holes in the bulk material, resulting in a high holes concentration which consequently causes a highly conductive p-type behavior in our Ge NCs films.

In order to investigate the transport behaviors of Ge NCs films, temperature-dependent conductivities were measured. It was found that the films annealed at 400 and $500^{\circ} \mathrm{C}$ exhibited a linear relationship of the $\ln \sigma$ versus $T^{-1}$ plot in the temperature region of 300-500 K in Figure 5, which matches the Arrhenius relationship between dark conductivity and temperature, described as the formula: $\sigma=\sigma_{0} \exp \left(-E_{a} / k_{B} T\right)$, where $\sigma_{0}$ is the preexponential factor of conductivity, $E_{a}$ is the conductivity activation energy, and $k_{B}$ is Boltzmann's constant. It is indicated that the carrier transport mechanism of Ge NCs films above room temperature is the thermal 


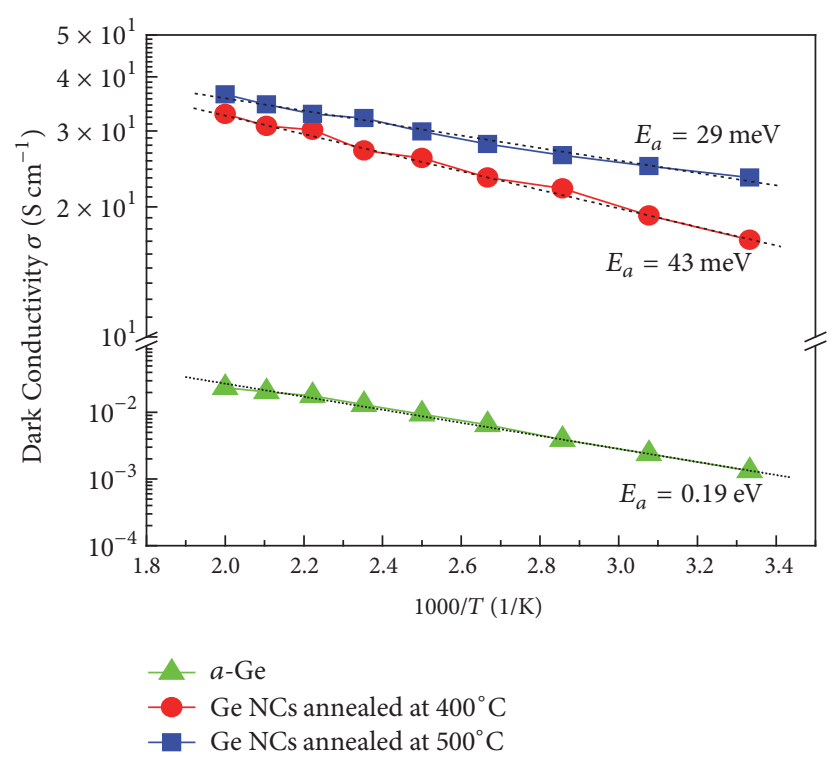

FIGURE 5: Temperature-dependent conductivities of all the samples in the temperature region of $300-500 \mathrm{~K}$.

activation conduction in the extended states [32]. The activation energy $E_{a}$ can be deduced through Arrhenius plots by the slope of the linear fit, which is related to the energy difference between the Fermi level and the top of valance band in p-type semiconductor [33]. The deduced conductivity activation energy $E_{a}$ of the Ge NCs films annealed at 400 and $500^{\circ} \mathrm{C}$ is 43 and $29 \mathrm{meV}$, respectively. Compared with $E_{a}$ of $190 \mathrm{meV}$ for the as-deposited film, we can conclude that the Fermi level is shifted toward the top of valance band and the more charge carriers are produced in Ge NCs films by thermal annealing, just as it has been illustrated earlier that surface states could give rise to the holes accumulation as well as the Fermi level shift.

We also measured the temperature-dependent conductivities in the low temperature range (10-300 K). However, the conductivity data obtained in the low temperature region cannot be well described by the Arrhenius relationship, which suggests different mechanisms dominating the carrier transport processes in Ge NCs films. In order to extract information about the transport behaviors of Ge NCs at low temperature, it is convenient to plot logarithm of the conductivity $(\ln \sigma)$ as a function of $T^{-1 / \alpha}$, in which $\alpha=1$ can be considered as an Arrhenius plot, and find a proper value of $\alpha$ which straightens out experimental curves. Consequently, the value of $\alpha$ was found to be around 2 from $120 \mathrm{~K}$ to $260 \mathrm{~K}$ and from $90 \mathrm{~K}$ to $230 \mathrm{~K}$ in the Ge NCs films annealed at 400 and $500^{\circ} \mathrm{C}$, respectively. Figure 6 shows $\ln \sigma$ plotted as a function of $T^{-1 / 2}$ of all the Ge NCs films. The solid lines are the results of the least squares fitting by straight lines. We can see that the observed $\alpha$ can be fitted very well with the straight lines. This temperature-dependent $\sigma$ behavior, which is usually expressed by $\sigma=\sigma_{0} \exp \left[-\left(T_{0} / T\right)^{1 / 2}\right]$, has been frequently observed in the similar works of $\mathrm{Ge}$ or $\mathrm{Si}$ nanocrystals $[34,35]$. This $\ln \sigma \propto T^{-1 / 2}$ behavior is frequently explained by the percolation-hopping model [36]. According

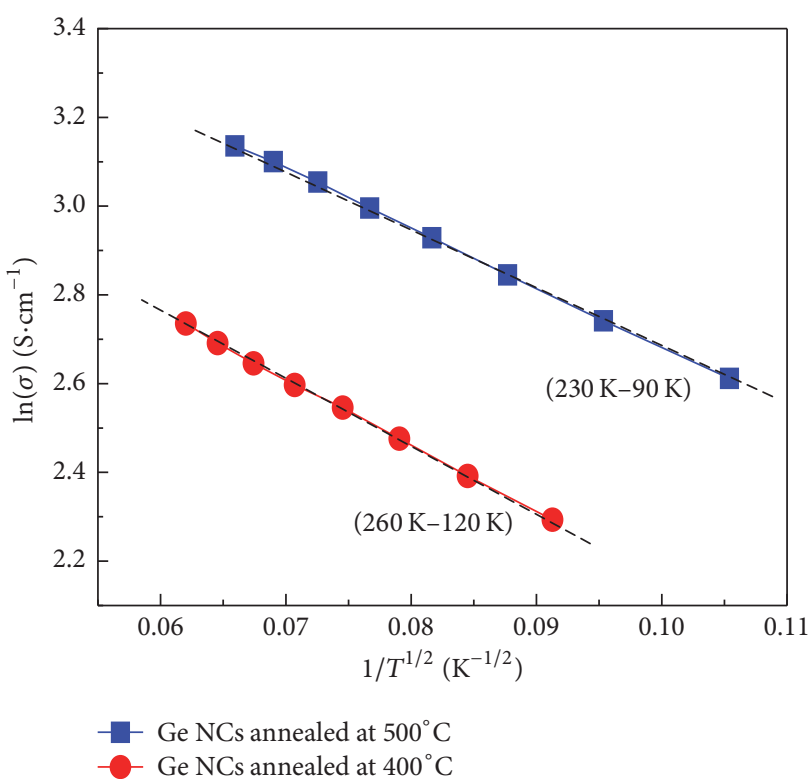

Figure 6: The $\ln \sigma$ plotted as a function of $T^{-1 / 2}$ of all Ge NCs films in the moderate temperature regions.

to this model, the conductive nanocrystals are dispersed in insulating matrices, which are separated from each other by a finite barrier. The thermally activated electrons can be transferred from one nanocrystal to another by tunneling the barrier. The percolation-hopping model can be suitable for the present samples because Ge NCs in our films are separated by the amorphous regions as can be seen in HRTEM images, and the current transport is considered to be made by the tunneling of electrons between the neighboring Ge NCs.

However, at the lower temperature $(<90 \mathrm{~K})$, the temperature-dependent behavior of conductivities was changed again and no longer dominated by the percolationhopping conduction mechanism. As shown in Figure 7, the $\ln \sigma \propto T^{-1 / 4}$ behavior was found in the Ge NCs films annealed at 400 and $500^{\circ} \mathrm{C}$, which indicates the Mott variable-range hopping (Mott-VRH) model following the formula: $\sigma=\sigma_{0} \exp \left[-\left(T_{0} / T\right)^{1 / 4}\right]$ [37]. It is reasonable since the ability of electrons for hopping between the neighboring Ge NCs is limited due to the freeze out of acoustic phonons as the temperature goes down to $90 \mathrm{~K}$. According to Mott's model, the lower temperature transport is due to the tunneling of carriers from occupied to unoccupied localized states [37]. The Mott-VRH conduction can be observed in the microcrystalline silicon ( $\mu c$-Si) films at low temperature as well [38-40]. Furthermore, it was found that, with the annealing temperature of Ge NCs films increasing, the temperature at which the conduction mechanism changes from $\ln \sigma \propto T^{-1 / 2}$ behavior (percolation-hopping process) to $\ln \sigma \propto T^{-1 / 4}$ behavior (Mott-VRH process) became lower as shown in Figure 7. For example, it is about $90 \mathrm{~K}$ for the $\mathrm{Ge}$ NCs film annealed at $400^{\circ} \mathrm{C}$ and decreased to $50 \mathrm{~K}$ for the Ge NCs film annealed at $500^{\circ} \mathrm{C}$. In the previous work, Fujii et al. have investigated the $\mathrm{Ge} \mathrm{NCs}$ embedded in $\mathrm{SiO}_{2}$ films. 


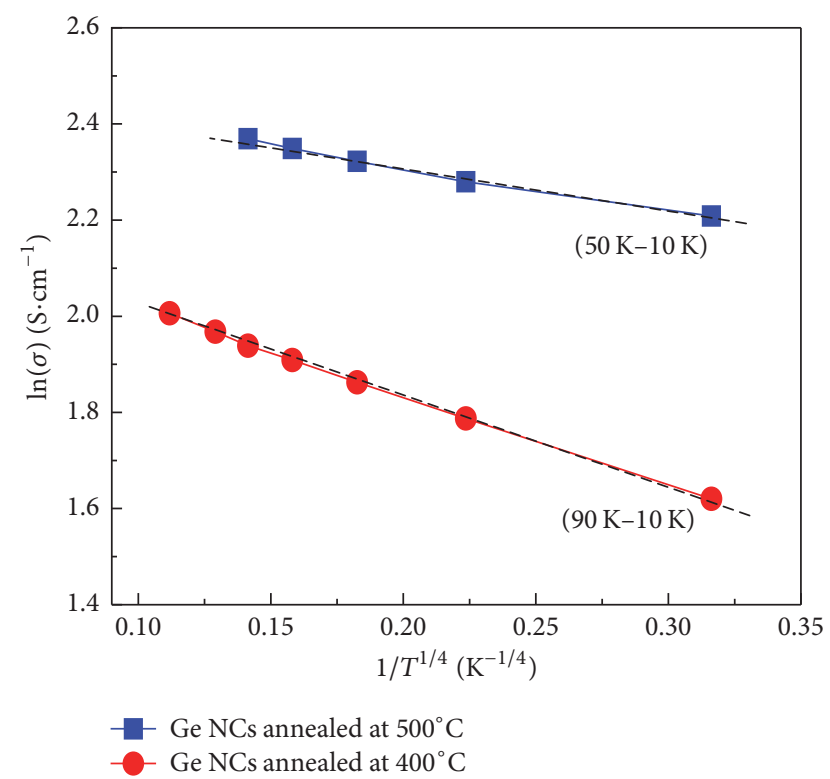

Figure 7: The $\ln \sigma$ plotted as a function of $T^{-1 / 4}$ of all Ge NCs films in the lower temperature regions.

They found that increasing the grain size had the effect of extending the $T^{-1 / 2}$ region to the lower temperature, which was explained by the decrease of the activation energy due to the growth of the nanocrystals [34]. Therefore, the crossover of $\ln \sigma \propto T^{-1 / 2}$ and $\ln \sigma \propto T^{-1 / 4}$ in our Ge NCs films shifting to the lower temperature is attributed to the increase of grain size in Ge NCs films caused by the increase of annealing temperature, which is also confirmed by Raman results.

\section{Conclusions}

In summary, Ge NCs films were fabricated by thermal annealing of the corresponding $a$-Ge:H films. It was found that both the crystallinity and average grain size in Ge NCs films were increased with the annealing temperature. Hall mobility reached the value of $182 \mathrm{~cm}^{2} / \mathrm{V}$.s in Ge NCs films due to the quality crystallization of films. Meanwhile, a high conductivity with p-type carrier type was achieved to the maximum of $25.6 \mathrm{~S} / \mathrm{cm}$ in Ge NCs films without any external doping, which is mainly attributed to the holes accumulation caused by the acceptor-like surface states. Carrier transport mechanisms in Ge NCs films were investigated by the temperature-dependent conductivities in the temperature region of $10-500 \mathrm{~K}$. Three kinds of conduction mechanisms were observed in different temperature regions. The thermal activation conduction in the extended states dominated the carrier transport process above $300 \mathrm{~K}$. Below room temperature, the carrier transport process was dominated by the percolation-hopping conduction below $260 \mathrm{~K}$ and turned to Mott-VRH conduction when the temperature falls below $90 \mathrm{~K}$. The temperature at which the conduction mechanism changes from the percolation-hopping behavior to the MottVRH behavior was decreased with the increase of grain size in Ge NCs films.

\author{
Abbreviations \\ a-Ge:H: Hydrogenated amorphous germanium \\ PECVD: Plasma enhanced chemical vapor deposition \\ Ge NCs: Ge nanocrystals \\ GB: $\quad$ Grain boundary \\ TEM: $\quad$ Transmission electron microscopy \\ Mott-VRH: Mott variable-range hopping.
}

\section{Conflicts of Interest}

The authors declare that they have no conflicts of interest.

\section{Authors' Contributions}

Dan Shan and Jun Xu conceived the idea and carried out the experiments. Dan Shan and Hongyu Wang participated in the preparation of the samples. Dan Shan, Hongyu Wang, Mingjun Tang, and Jun Xu took part in the experiments and the discussion of the results. Dan Shan drafted the manuscript with the instruction of Hongyu Wang, Mingjun Tang, and Jun $\mathrm{Xu}$. All authors read and approved the final manuscript.

\section{Acknowledgments}

This work was supported by NSFC [Grant no. 61735008 and Grant no. 11774155]; Natural Science Foundation of Yangzhou City [Grant no. YZ2016123]; Special Fund for City School Cooperation of Yangzhou City [Grant no. SCX2017020022]; NUPTSF [Grant no. NY218094] "Qing Lan project" of the Higher Education Institutions of Jiangsu Province; and "Qing Lan project" of Yangzhou Polytechnic Institute.

\section{References}

[1] C. Bonafos, Y. Spiegel, P. Normand et al., "Controlled fabrication of $\mathrm{Si}$ nanocrystal delta-layers in thin $\mathrm{SiO}_{2}$ layers by plasma immersion ion implantation for nonvolatile memories," Applied Physics Letters, vol. 103, no. 25, Article ID 253118, 2013.

[2] X. Xu, Y. Cao, P. Lu, J. Xu, W. Li, and K. J. Chen, "Electroluminescence Devices Based on Si Quantum Dots/SiC Multilayers Embedded in PN Junction," IEEE Photon. J, vol. 6, Article ID 2200207, 2014.

[3] Y. Q. Cao, P. Lu, X. W. Zhang, J. Xu, L. Xu, and K. J. Chen, "Enhanced photovoltaic property by forming p-i-n structures containing Si quantum dots/SiC multilayers," Nanoscale Res. Lett, vol. 9, pp. 34-40, 2014.

[4] F. Prido, T. Gregorkiewicz, M. Galli, and T. F. Krauss, "Silicon nanostructures for photonics and photovoltaics," Nat. Nanotechnol, vol. 9, pp. 19-32, 2014.

[5] W. W. Mu, P. Zhang, J. Xu, SH. Sun, and W. Li, "Direct-current and alternating-current driving si quantum dots-based light emitting device," IEEE J. Sel. Top. Quant, vol. 20, pp. $8200206-$ 8200212, 2013.

[6] M. Mitsui, K. Arimoto, J. Yamanaka, K. Nakagawa, K. Sawano, and Y. Shiraki, "Influence of Ge atoms on mobility and junction properties of thin-film transistors fabricated on solid-phase crystallized poly-SiGe," Applied Physics Letters, vol. 89, no. 19, Article ID 192102, 2006. 
[7] P.-Y. Kuo, T.-S. Chao, J.-S. Huang, and T.-F. Lei, "Poly-Si thinfilm transistor nonvolatile memory using Ge nanocrystals as a charge trapping layer deposited by the low-pressure chemical vapor deposition," IEEE Electron Device Letters, vol. 30, no. 3, pp. 234-236, 2009.

[8] C.-C. Wang, D.-S. Wuu, S.-Y. Lien et al., "Characterization of nanocrystalline SiGe thin film solar cell with double gradeddead absorption layer," International Journal of Photoenergy, vol. 2012, Article ID 890284, 2012.

[9] W.-T. Lai, P.-H. Liao, A. P. Homyk, A. Scherer, and P.-W. Li, "SiGe quantum dots over Si pillars for visible to near-infrared broadband photodetection," IEEE Photonics Technology Letters, vol. 25, no. 15, pp. 1520-1523, 2013.

[10] C. Song, G. R. Chen, J. Xu et al., "Evaluation of microstructures and carrier transport behaviors during the transition process from amorphous to nanocrystalline silicon thin films," Journal of Applied Physics, vol. 105, no. 5, Article ID 054901, 2009.

[11] L. R. Wienkes, C. Blackwell, and J. Kakalios, "Electronic transport in doped mixed-phase hydrogenated amorphous/ nanocrystalline silicon thin films," Applied Physics Letters, vol. 100, no. 7, Article ID 072105, 2012.

[12] D. Shan, Y. Ji, J. Xu et al., "Microstructure and carrier-transport behaviors of nanocrystalline silicon thin films annealed at various temperatures," Physica Status Solidi (a) - Applications and Materials Science, vol. 213, no. 7, pp. 1675-1679, 2016.

[13] D. Shan, Y. Ji, D. Li et al., "Enhanced carrier mobility in Si nanocrystals via nanoscale phosphorus doping," Applied Surface Science, vol. 425, pp. 492-496, 2017.

[14] M. Fujii, Y. Inoue, S. Hayashi, and K. Yamamoto, "Hopping conduction in $\mathrm{SiO} 2$ films containing $\mathrm{C}, \mathrm{Si}$, and Ge clusters," Applied Physics Letters, vol. 68, no. 26, pp. 3749-3751, 1996.

[15] M. A. Rafiq, Y. Tsuchiya, H. Mizuta et al., "Charge injection and trapping in silicon nanocrystals," Applied Physics Letters, vol. 87, no. 18, Article ID 182101, pp. 1-3, 2005.

[16] M. A. Rafiq, Y. Tsuchiya, H. Mizuta et al., "Hopping conduction in size-controlled Si nanocrystals," Journal of Applied Physics, vol. 100, no. 1, Article ID 014303, 2006.

[17] C. Peng, K. D. Hirschman, and P. M. Fauchet, "Carrier transport in porous silicon light-emitting devices," Journal of Applied Physics, vol. 80, no. 1, pp. 295-300, 1996.

[18] H. Grabert and M. H. Devoret, NATO ASI Series B, Plenum Press, 1991.

[19] P. R. Poulsen, M. Wang, J. Xu et al., "Role of hydrogen surface coverage during anodic plasma deposition of hydrogenated nanocrystalline germanium," Journal of Applied Physics, vol. 84, no. 6, pp. 3386-3391, 1998.

[20] P. Caldelas, A. G. Rolo, M. J. M. Gomes et al., "Raman and XRD studies of Ge nanocrystals in alumina films grown by RFmagnetron sputtering," Vacuum, vol. 82, no. 12, pp. 1466-1469, 2008.

[21] X. J. Hao, E.-C. Cho, C. Flynn, Y. S. Shen, G. Conibeer, and M. A. Green, "Effects of boron doping on the structural and optical properties of silicon nanocrystals in a silicon dioxide matrix," Nanotechnology, vol. 19, no. 42, Article ID 424019, 2008.

[22] S. Mirabella, R. Agosta, G. Franzó et al., "Light absorption in silicon quantum dots embedded in silica," Journal of Applied Physics, vol. 106, no. 10, Article ID 103505, 2009.

[23] D. Das and K. Bhattacharya, "Characterization of the Si:H network during transformation from amorphous to micro- and nanocrystalline structures," Journal of Applied Physics, vol. 100, no. 10, p. 103701, 2006.
[24] Y. Gao, X. Pi, X. Wang et al., "Structures, oxidation, and charge transport of phosphorus-doped germanium nanocrystals," Particle \& Particle Systems Characterization, vol. 33, no. 5, pp. 271278, 2016.

[25] B. Zhang, S. Shrestha, M. A. Green, and G. Conibeer, "Surface states induced high P-type conductivity in nanostructured thin film composed of Ge nanocrystals in SiO2 matrix," Applied Physics Letters, vol. 97, no. 13, Article ID 132109, 2010.

[26] B. Zhang, S. Shrestha, M. A. Green, and G. Conibeer, "Size controlled synthesis of $\mathrm{Ge}$ nanocrystals in $\mathrm{SiO}_{2}$ at temperatures below $400^{\circ} \mathrm{C}$ using magnetron sputtering," Applied Physics Letters, vol. 96, no. 26, Article ID 261901, 2010.

[27] S. Zhang, E. R. Hemesath, D. E. Perea, E. Wijaya, J. L. LenschFalk, and L. J. Lauhon, "Relative influence of surface states and bulk impurities on the electrical properties of ge nanowires," Nano Letters, vol. 9, no. 9, pp. 3268-3274, 2009.

[28] J.-S. Park, B. Ryu, C.-Y. Moon, and K. J. Chang, "Defects responsible for the hole gas in Ge/Si core-shell nanowires," Nano Letters, vol. 10, no. 1, pp. 116-121, 2010.

[29] A. Dimoulas, P. Tsipas, A. Sotiropoulos, and E. K. Evangelou, "Fermi-level pinning and charge neutrality level in germanium," Applied Physics Letters, vol. 89, no. 25, Article ID 252110, 2006.

[30] R. H. Kingston, "Review of germanium surface phenomena," Journal of Applied Physics, vol. 27, no. 2, pp. 101-114, 1956.

[31] P. Tsipas and A. Dimoulas, "Modeling of negatively charged states at the Ge surface and interfaces," Applied Physics Letters, vol. 94, no. 1, Article ID 012114, 2009.

[32] S. Y. Myong, K. S. Lim, and M. Konagai, "Effect of hydrogen dilution on carrier transport in hydrogenated boron-doped nanocrystalline silicon-silicon carbide alloys," Applied Physics Letters, vol. 88, no. 10, Article ID 103120, 2006.

[33] C. Song, Y. Rui, Q. Wang et al., "Structural and electronic properties of $\mathrm{Si}$ nanocrystals embedded in amorphous $\mathrm{SiC}$ matrix," Journal of Alloys and Compounds, vol. 509, no. 9, pp. 3963-3966, 2011.

[34] M. Fujii, O. Mamezaki, S. Hayashi, and K. Yamamoto, "Current transport properties of SiO2 films containing Ge nanocrystals," Journal of Applied Physics, vol. 83, no. 3, pp. 1507-1512, 1998.

[35] X. Zhou, K. Usami, M. A. Rafiq, Y. Tsuchiya, H. Mizuta, and S. Oda, "Influence of nanocrystal size on the transport properties of Si nanocrystals," Journal of Applied Physics, vol. 104, no. 2, Article ID 024518, 2008.

[36] E. Šimánek, "The temperature dependence of the electrical resistivity of granular metals," Solid State Communications, vol. 40, no. 11, pp. 1021-1023, 1981.

[37] N. F. Mott, "Conduction in non-crystalline materials," Philosophical Magazine, vol. 19, no. 160, pp. 835-852, 1969.

[38] R. Brenot, R. Vanderhaghen, B. Drévillon, P. Roca I Cabarrocas, R. Rogel, and T. Mohammed-Brahim, "Transport mechanisms in hydrogenated microcrystalline silicon," Thin Solid Films, vol. 383, no. 1-2, pp. 53-56, 2001.

[39] F. Liu, M. Zhu, Y. Feng, Y. Han, and J. Liu, “Electrical transport properties of microcrystalline silicon thin films prepared by Cat-CVD," Thin Solid Films, vol. 395, no. 1-2, pp. 97-100, 2001.

[40] G. Ambrosone, U. Coscia, A. Cassinese et al., "Low temperature electric transport properties in hydrogenated microcrystalline silicon films," Thin Solid Films, vol. 515, no. 19, pp. 7629-7633, 2007. 


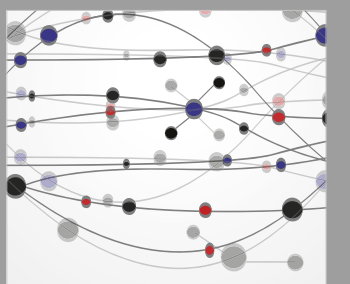

The Scientific World Journal
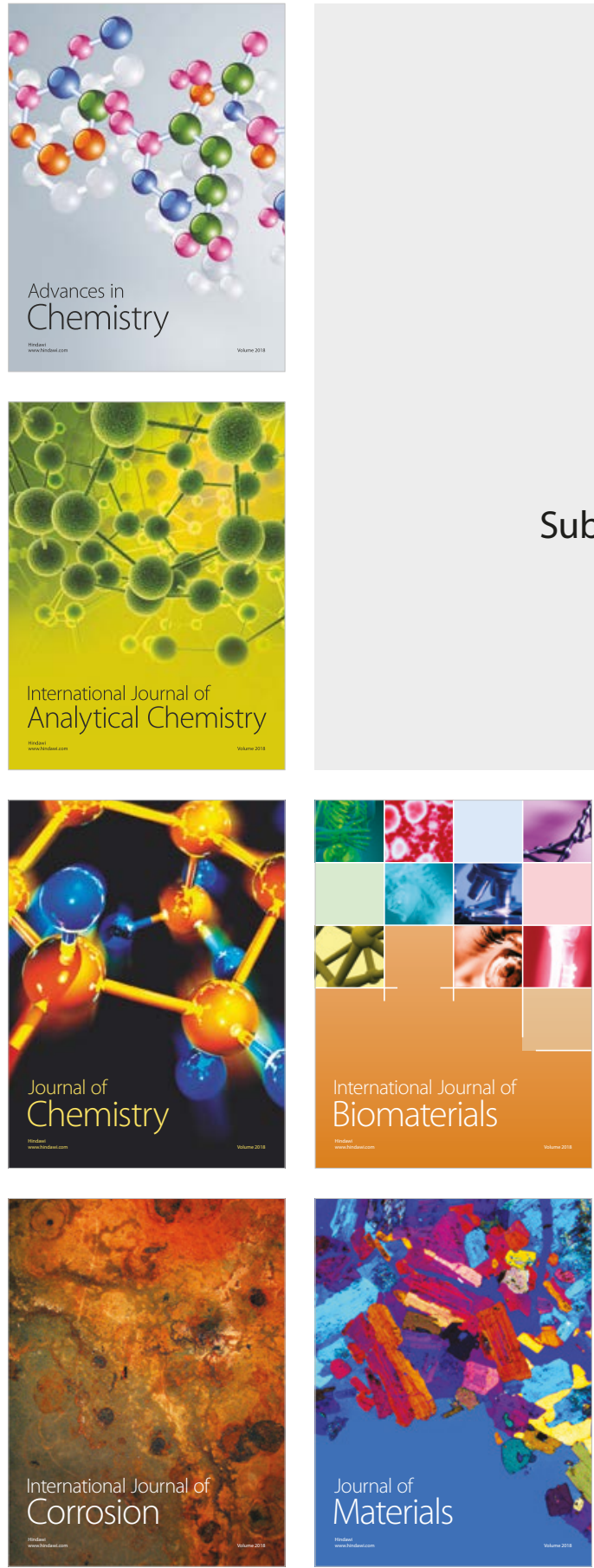

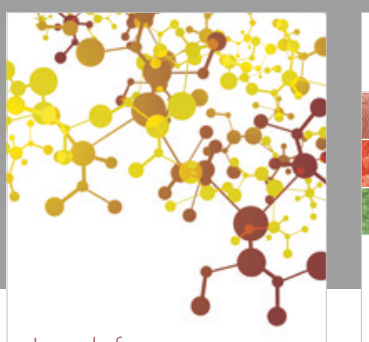

Journal of

Applied Chemistry
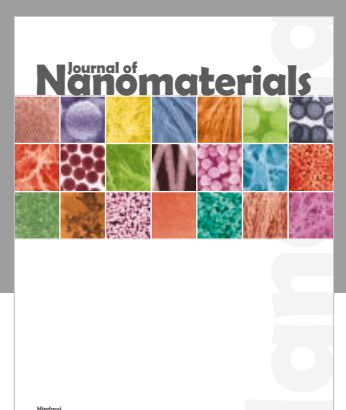

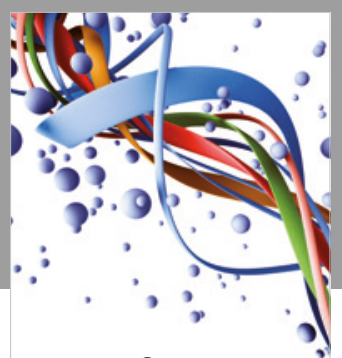

Scientifica

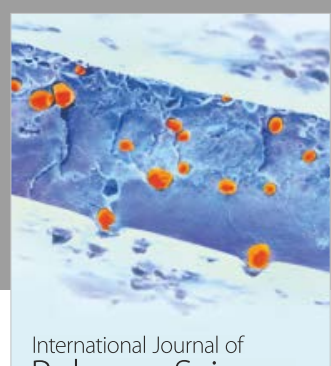

Polymer Science

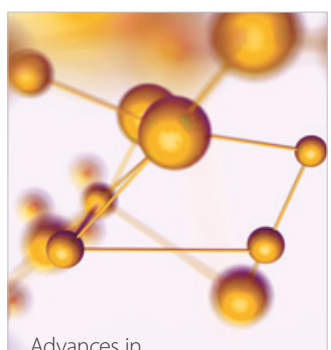

Physical Chemistry
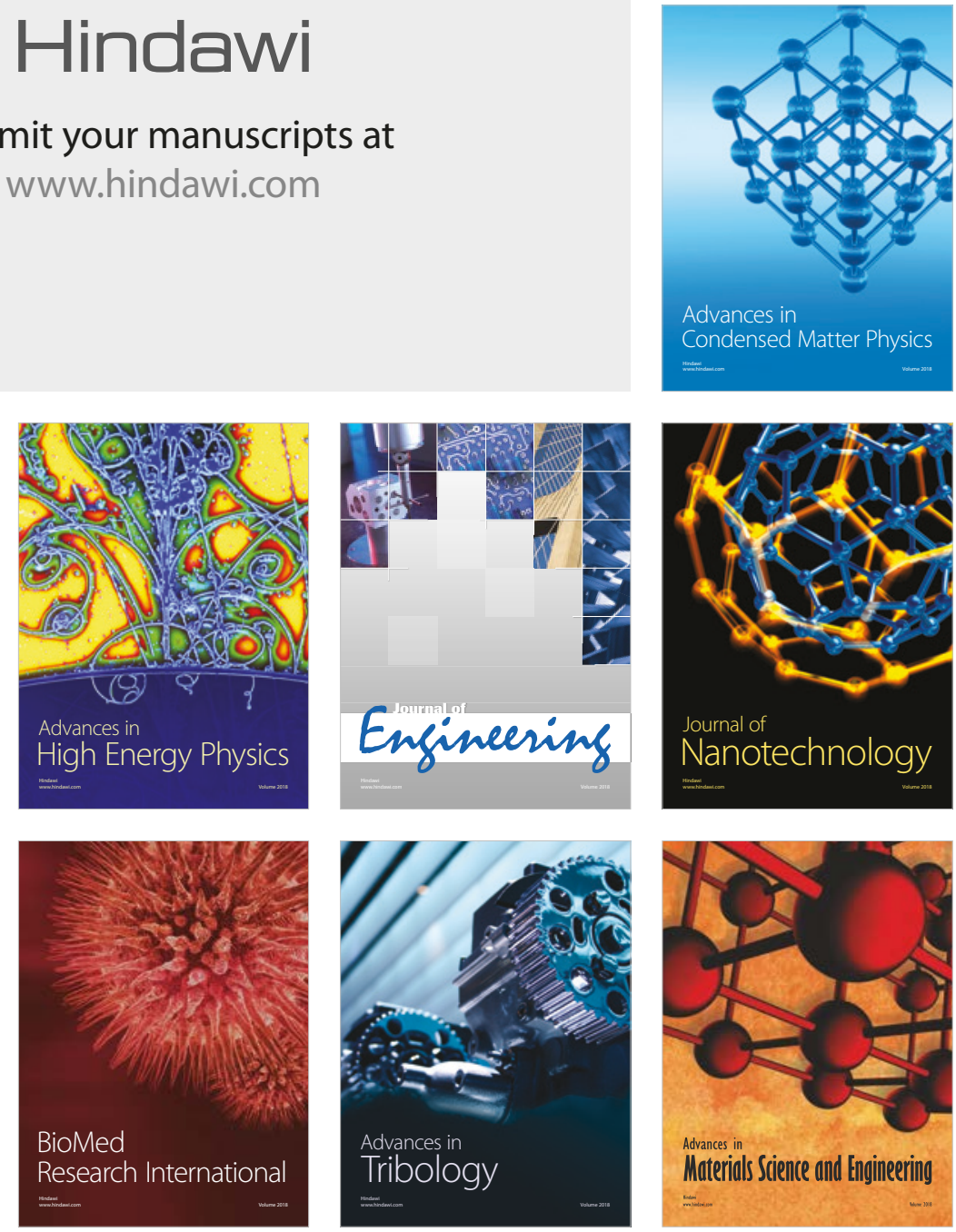